\title{
Could Resilience and Flourishing be Mediators in the Relationship between Mindfulness and Life Satisfaction for Saudi College Students? A Psychometric and Exploratory Study
}

\author{
Marei, S. Younes* \\ Helwan University, Egypt
}

Mohammed R. Alzahrani

Umm Alqura University

Kingdom of Saudi Arabia

Received: 9/12/2017 Accepted: 26/4/2018

\begin{abstract}
In There are few Arabic studies examining the disposition of mindfulness, resilience, and well-being of college students. The purpose of the present study is to examine the factor structure and internal consistency of the Arabic version of mindful attention awareness scale (MAAS) and the brief resilience scale, and to test a model that describes the role of resilience and well-being, in relation to mindfulness and life satisfaction. For this reason, a study of two folds was conducted with the assistance of Umm Al-Quraa University students $(n=562 ; n=$ 534). Mindfulness, resilience, flourishing and life satisfaction scales are the variables that were used. Results show that both mindfulness and resilience scales were revealed to be unidimensional and have high reliability estimates. Both resilience and flourishing are partial mediators in the relationship between mindful and life satisfaction. In addition, resilience had an impact on flourishing. Mindfulness partially had an effect on life satisfaction. In conclusion, mindfulness and resilience scales had good structural validity and reliability.
\end{abstract}

Keywords: Mindfulness, resilience, well-being, life satisfaction.

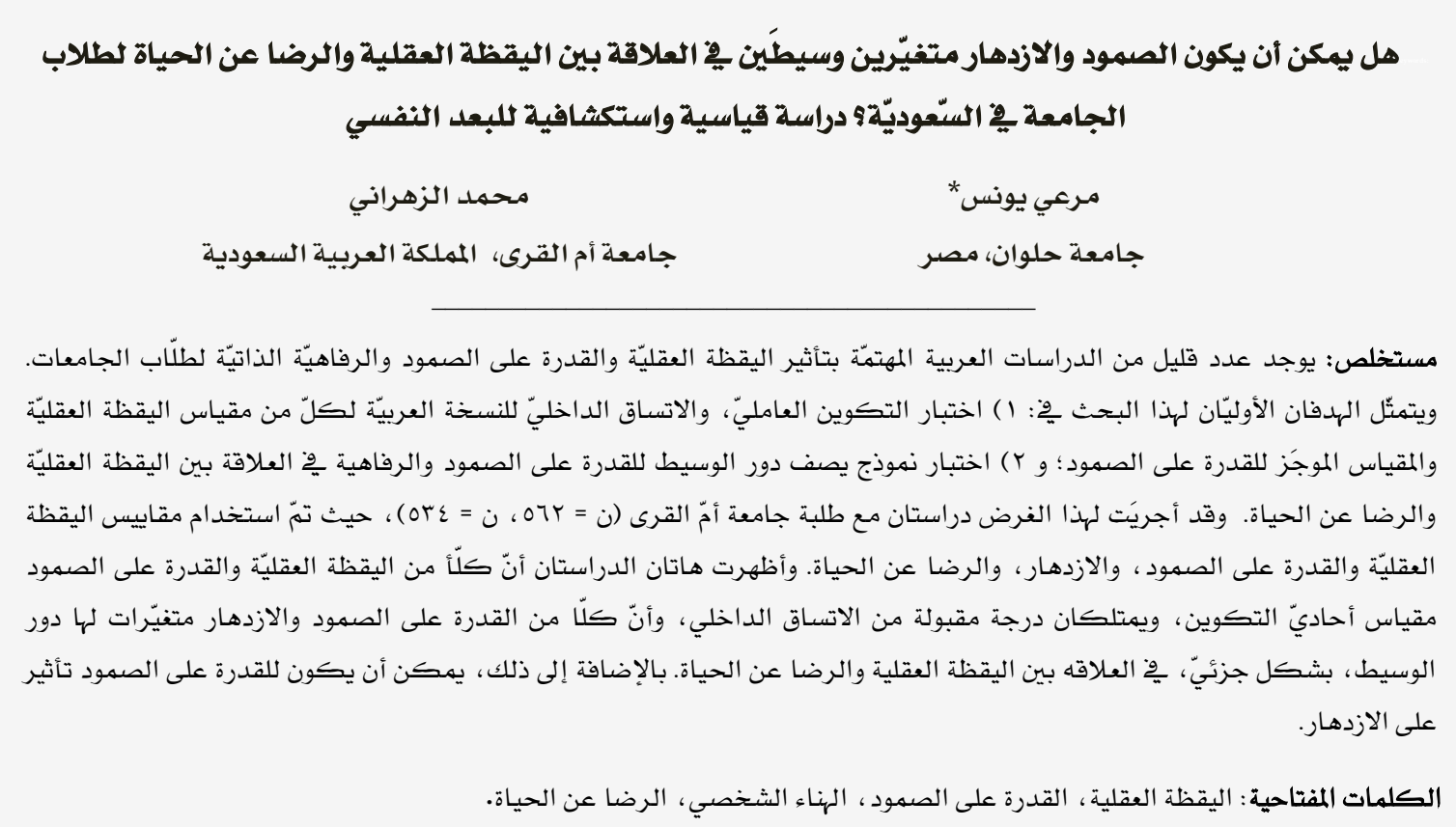

*msalamayounes@hotmail.fr 
Mindfulness, resilience, flourishing and life satisfaction have been studied in-depth with different settings and models. However, in Arabic literature, the researchers did not find any studies that investigated the relationships among mindfulness, resilience, flourishing and life satisfaction, particularly among college students.

Brown and Ryan (2003) defined mindfulness as a flexible state of mind consciousness of an individual surrounding with polished attention and non-evaluative awareness of one's internal and external experiences. They defined it as a psychological trait that refers to the tendency of mindfulness in everyday life. Mindfulness is a practice of paying attention to the present moment in a purposeful and nonjudgmental manner (Kabat-Zinn, 2009). Another conceptualization argues that mindfulness is a state of awareness of oneself and the environment without judging or reacting non-intentionally while involved in describing one's subjective experience (Baer, Smith, Hopkins, Krietemeyer, \& Toney, 2006). Mindfulness may also be improved through meditation or psychological training (Baer et al., 2006). Moreover, mindfulness can be improved through resilience as mindful people are better able to respond to difficult situations; they tend to be more creative and easily cope with difficult thoughts and emotions without becoming overwhelmed or aggressive (Langer \& Moldoveanu, 2000).

Mindfulness can be considered as an enhanced attention and awareness of current experience or present reality. Specifically, a core characteristic of mindfulness has been described as an open or receptive awareness and attention (Martin, 1997), which may be reflected in a more regular or sustained state of mind consciousness with respect to ongoing events and experiences. Awareness or attention can also be divided, such as when people are engaged in doing multiple tasks or preoccupied with concerns that reduce the quality of engagement with what is focally present. Mindfulness is also distracted when individuals compulsively or automatically get disturbed, without awareness of or attention to one's behavior (Deci \& Ryan, 1980). Finally, mindlessness, is a relative absence of mindfulness that can be defensively motivated when an individual refuses to acknowledge or attend to a thought, emotion, motive, or object of perception. These forms of consciousness thus serve as concrete counterpoints to the mindful person with presence and the attention given to current experience within and without oneself that such presence entails. As presently defined, mindfulness bears some relation to other constructing elements that have received empirical attention.

Resilience is defined as a personal trait that helps individuals to overcome adversity and achieve good adjustment and development during annoying circumstances. It is a trait that inoculates individuals against the impact of adversity and traumatic events (Ong, Bergeman, Bisconti, \& Wallace, 2006). It is the competence to deal with stress or trauma and to 'bounce-back from adversity with a varying degree of severity over the lifetime. Resources for resilience can be found in the individual or in its environment where he/she operates their routine actions (Windle, Bennett, \& Noyes, 2011). One key factor to promote resilience is temperance, which is the main character of strength that includes forgiveness and mercy. Resilience is associated with less severe reactions to negative events, less aggression, and keeness in making new relationships (Cohrs, Christie, White, \& Das, 2013).

Several reasons can be found which show the importance of using resilience by incorporating youth as a study sample. For example, more than half of human beings experience at least one traumatic event during their lifetime, but most of them recover without experiencing mental illness (Windle et al., 2011). To the contrary, $30 \%$ to $90 \%$ of people experiencing such traumatic events report that there should be an amplified change in quality of life (Aspinwall \& MacNamara, 2005). For achieving positive results after stressful events, resilience is considered an important factor (Catalano, Chan, Wilson, Chiu, \& Muller, 2011). It delivers positive a effect on the rehabilitation of different illnesses, both physical and mental such as breast cancer (Markovitz, Schrooten, Arntz, \& Peters, 2015). Resilience protects and prevents individuals from clinical psychopathology (Portzky, Wagnild, De Bacquer, \& Audenaert, 2010). Highly resilient people often show reactions to traumatic events, for example being upset, but those reactions are rather short-termed. Additionally, 
highly resilient people return faster than to non-resilient people to their actual level of functioning. Less resilient people can also recover from traumatic events, but usually, this recovery is characterized as a lack of functioning in everyday life (Portzky et al., 2010).

Because of these reasons, resilience is becoming increasingly important in different professional fields, such as clinical psychology and medical sciences (Portzky et al., 2010). Finding an adequate measurement of resilience can further improve work related activities in different spheres of both professional and personal fields. For that reason, resilience and related factors should be studied thought this respective examination.

With a high level of resilience, many people experience a lower level of generalized emotional distress, depression, and anxiety. Those people have higher levels of positive affectivity that can be perceived as well-being and have high acceptance of disability that were reported in this study. More resilient people were found to experience less pain and resilience has been linked to posttraumatic growth (Consten, 2016). Altogether, resilience is associated with more effective coping-strategies, better effects in therapy and limited ratio of comiting suicidal attempts (Portzky et al., 2010).

Flourishing is the ultimate end-state in psychology and served as a key concept in the field of positive psychology and its related research domains. For evaluating the flourishing state, there are four different operationalization: 1) Keyes (2005), 2) Diener et al. (2010), Huppert and So (2013) models (Hone, Jarden, Schofield, \& Duncan, 2014). In the present research, we adopted the models of Keyes (2005) and Diener et al., (2010).

Flourishers are those individuals with both high levels of hedonic well-being and eudemonic well-being. Although many researchers have focused on one or another of these domains, only few have investigated the comprehensive state of flourishing (SchotanusDijkstra et al., 2016). Flourishing is a measure of overall life well-being and is viewed as an important idea of happiness (Fredrickson \& Losada, 2005). Many components and concepts contributed to the overall concept of flourishing and the benefits of a life that can be characterized as flourishing (Huppert \& So,
2013). The emerging field of positive psychology aims to redress this particular imbalance. In flourishing, different scholars applied their scientific analyses in order to study the good life, expanding the scope of social and psychological research to include happiness, wellbeing, courage, citizenship, play, and the satisfaction of healthy work and healthy relationships. Their findings reveals that a sense of meaning and a feeling of richness emerge in life as people submerge themselves in activities, relationships, and the pursuit of intrinsically satisfying goals like overcoming adversity or serving one's community through volunteering (Keyes, 2010). A conceptual framework is offered which equates high level of wellbeing with positive mental health. Well-being is seen on the opposite end of a spectrum which leads towards mental disorders such as depression and anxiety. By examining internationally agreed criteria for depression and anxiety (DSM and ICD classifications), and defining the opposite of each symptom, we identify ten features of positive well-being. These combine feeling and functioning, i.e. hedonic and eudaimonic aspects of well-being: competence, emotional stability, engagement, meaning, optimism, positive emotion, positive relationships, resilience, self-esteem, and vitality (Huppert \& So, 2013).

From Keyes' view, flourishing generally refers to the good life; feeling good and functioning of human actions with great effectiveness (Huppert \& So, 2013). Flourishing is a concept that helps social scientists and psychologists to study and measure the variables of fulfillment, purpose, meaning, and happiness. Many authors then claim that flourishing is the product for adults who have healthy mentally with a high level of emotional well-being which demonstrates happiness and satisfaction of life. Flourishing people tend to feel some degree of mastery environment, have a life purpose, accept all parts by themselves, experience personal growth and have more autonomy (Haidt \& Keyes, 2003).

The Flourishing Scale (FS) is a measure of psychosocial well-being that complements existing measures of subjective well-being. Authors created this brief measure to capture major aspects of this type of "prosperity" based on recent psychological theories of human flourishing. Based on earlier humanistic psychology theories, Ryff and Keyes, 1995; Ryff and 
Singer, (1998) suggest that there are several universal human psychological needs, such as the need for competence, relatedness, and selfacceptance. Some of these factors are assessed by the Flourishing Scale (Diener, Wirtz, et al., 2010).

According to Diener, Emmons, Larsen, and Griffin (1985), there are three separable components of subjective well-being: positive affect (PA), negative affect (NA), and life satisfaction. The determinants of well-being and life satisfaction are highly individualized and also personalized. It all depends on an individual and their own value orientations (Prasoon \& Chaturvedi, 2016). Whereas the effective part is a hedonic estimation guided by emotions and feeling the cognitive part is an information-based assessment of one's life for which people judge the extent that their life so far measures up to their expectations and bears a resemblance to their envisioned ideal life (Van Hoorn, 2007).

Life satisfaction is an overall assessment of feelings and attitudes about one's life at a particular point in time rang from negative to positive life experience. It is one of the three major indicators of well-being that are life satisfaction, positive effect, and negative effect (Diener, 1994). Life satisfaction is the degree to which a person positively evaluates the overall quality of his/her life as a whole. It is believed to have antecedents in the work domain, family domain, and personality traits (Veenhoven, 1996). Subjective well-being: Much of the current literature on well-being uses terms like happiness, life satisfaction, and subjective well-being interchangeably, although this is not completely accurate (Frey \& Stutzer, 2002; Lu, 2010; Senik, 2011; Wright \& Cropanzano, 2000). Life satisfaction is the more general construct of subjective well-being (SWB). Theory and research from outside of the rehabilitation fields have suggested that SWB has at least three components: positive affective appraisal, negative affective appraisal, and life satisfaction. Life satisfaction is distinguished from an affective appraisal because it is more cognitively associated with mind than emotionally driven. Life satisfaction can be assessed specifically with a particular domain of life (e.g., work, family) or globally (Diener \& Diener, 2009).
Life satisfaction is attributable to a family of personality, genetic, and social-cognitive factors such as goal-directed activity, selfefficacy, outcome expectations, and environmental support (Buetell, 2006). Income and individual happiness became an important part of the study on life satisfaction as suggested by number of theorists like Di Tella, Haisken-De New, and MacCulloch, (2010); Diener, Kahneman, and Helliwell, (2010); Frijters, Johnston, and Shields, (2011).

\section{Mindfulness, Resilience, Well-being and Life Satisfaction}

A review of the literature revealed an association between mindfulness and resilience. It can be taught through psychological interventions, as Van Breda (2001) argues that mindfulness-based training may be an efficacious intervention in order to increase resilience (Diener, Wirtz, et al., 2010; Lightsey Jr, 2006; Schueller \& Parks, 2014). In addition, Jha, Stanley, Kiyonaga, Wong, and Gelfand (2010) supported the notion of a relationship between mindfulness and resilience. These authors showed that mindfulness-based fitness training (MMFT) delivered potential benefits and is also an effective method of increasing both resilience and psychological well-being of soldiers whose deployment and occupation increases the risk of psychological trauma. Further, support for an association between resilience and mindfulness was found by Chavers (2013) who suggests a significant correlation between mindfulness and resilience with mindfulness categorized as significant predictor of resilience.

Recent research has established the effect of mindfulness on well-being and life satisfaction by investigating the potential mediating role of resilience which has an impact on mindfulness, life satisfaction and subjective wellbeing. The structural equation modeling (SEM) results showed that resilience partially mediated by the relationship between mindfulness, life satisfaction and affect components. The findings support the significant role of resilience in mindfulness employing its effects. This study contributes to the possible mechanism of the association between mindfulness and subjective well-being (Bajaj \& Pande, 2016). More precisely, correlational research has demonstrated that measures of trait mindfulness are closely associated with 
higher levels of subjective well-being (Carmody \& Baer, 2008; Schutte \& Malouff, 2011; Weinstein, Brown, \& Ryan, 2009). It is firmly believed by previous scholars that an increase in mindfulness through interventions such as meditation training, results in the increase of individuals' well-being (Cohen \& Miller, 2009; Grossman, Tiefenthaler-Gilmer, Raysz, \& Kesper, 2007; Huppert \& Johnson, 2010).

In the same direction, some studies conducted on college-level students have shown that mindfulness may influence well-being through mediators such as emotional intelligence, core self-evaluation and self-esteem (Schutte \& Malouff, 2011; Tsaousis, Nikolaou, Serdaris, \& Judge, 2007). Nevertheless, this model is rather unacceptable due to its limited ability to explain mindfulness's beneficial effects on well-being since empirical evidence is in support of the partial mediating role of these mediators. The expectations of other possible mediators, such as resilience, can explain the nature of the relationship between mindfulness and subjective well-being. In this study, we attempt to explore the mediating role of resilience with the impact of mindfulness on life satisfaction and affect as indices of subjective well-being.

\section{The present research}

Previous studies show a strong relationship between mindfulness, resilience and life satisfaction (Bajaj \& Pande, 2016; Pidgeon \& Keye, 2014). Mindfulness practices have been shown to have a positive impact on life satisfaction (Hu, Zhang, \& Wang, 2015; Kong, Wang, Hu, \& Liu, 2015). Mindfulness also has an impact on flourishing and well-being (Akin \&Akin, 2015; Schutte \& Malouff, 2011; Shapiro, Oman, Thoresen, Plante, \& Flinders, 2008). Mindfulness has a greater effect on job satisfaction (Fortney, Luchterhand, Zakletskaia, Zgierska, \& Rakel, 2013; Hülsheger, Alberts, Feinholdt, \& Lang, 2013) and romantic and marital satisfaction (Barnes, Brown, Krusemark, Campbell, \& Rogge, 2007). However, only limited research has studied the effect of mindfulness on satisfaction for college students (Collard, Avny, \& Boniwell, 2008).

Concerning the relationship between resilience and life satisfaction, many researchers confirm this relation in work assessment (Collins, 2007; Matos, Neushotz, Griffin, \& Fitzpatrick, 2010) and in the academic context (Abolghasemi \& Varaniyab, 2010; Collins, 2007). Concerning resilience and well-being, structural equation modeling analyses (Burns, Anstey, \& Windsor, 2011; Mak, Ng, \& Wong, 2011) showed that resilience and well-being have a strong relationship. Resilience has an impact on wellbeing and happiness. In different countries and cultures, many researchers have shown a high level of school engagement that is positively associated with academic success and students' well-being, such as positive emotions and life satisfaction (Upadyaya \& SalmelaAro, 2013; Van Zyl \& Rothmann, 2012). Structural equation modeling (SEM) showed that resilience partially mediated the relationship between mindfulness and life satisfaction. The findings corroborate an important role of resilience in mindfulness, exerting its beneficial effects. This study makes a contribution to the potential mechanism of the association between mindfulness and subjective well-being of college students that are based in Kingdom of Saudi Arabia.

More recently, relationships among mindfulness, resilience, and well-being were shown (Pidgeon \& Keye, 2014). Little information, however, is known about the relationships among mindfulness, resilience, well-being and life satisfaction. According to previous studies, mindfulness, resilience, and well-being are directly associated with each other. However, we have no information about the direction of these relationships. In other words, could resilience predict well-being? Or do resilience and well-being have the same level and could they predict another dependent variable? No study has been conducted to predict life satisfaction by studying mindfulness, resilience, flourishing particularly for Arab college students. In sum, studies using path analysis to explain these relationships among mindfulness, resilience, well-being and life satisfaction are still very rare.

In the Arabic literature, we did not find any study examining the relationships among mindfulness, resilience, flourishing and life satisfaction for college students. Only a correlational study between resilience and wellbeing for addictive and non-addictive students has been published (Manakhreh \& Yahya, 2017). There are several reasons for limited existing literature on the mindfulness and resilience. One essential contributor is that in 
the Arabic language, there is a shortage or unavailability of valid and reliable scales to measure either mindfulness or resilience. More precisely, we have translated scales in Arabic but we did not find any valid and reliable questionnaires measuring the two variables. For that reason, the first study must be conducted to Arabize and validate the mindfulness and resilience scales. The second was to explore the relationships among mindfulness, resilience, flourishing and satisfaction with life. Flourishing and life satisfaction scales were already translated and validated in Arabic (Abdallah, 1998; Salama-Younes, 2017).

The specific goals of the present research were threefold. It was hypothesized that both of the Arabic version of mindfulness and resilience scales are based on a one-dimensional factor. As aresult, confirmatory factor analysis (CFA) will be performed. Although the relationships among mindfulness, resilience and wellbeing/flourishing have been explored in previous research, they are not conducted on Arabic College Students (Bajaj \& Pande, 2016; Montero-Marin, Tops, Manzanera, Demarzo, de Mon, \& García-Campayo, 2015), the path of relationships for these variables with Arabic speaking countries has not been explored yet. Bajaj and Pande (2016) showed that resilience partially mediated the relationship between mindfulness and well-being as measured by life satisfaction and affect components. In sum, could resilience be a mediator between mindfulness and well-being? Could resilience and well-being be mediator variables in the relationships between mindfulness and life satisfaction? Two path analysis models have been used to explore these relationships. The objective of the first study was to test the structural validity of the Arabic versions. The paper version was administered just after finishing their course session.

\section{Study 1}

\section{Method}

\section{Participants and procedure}

To test the structural validity using the CFA, a sample was taken from Umm Al Quraa University that responded to these two scales. Students were all from the Abidiyyah campus. They all were undergraduate students and studied in one of the following six colleges: education, social sciences, applied sciences, computer and information systems, medicine, and applied medical science. The distribution of the sample in these colleges was classified into three different domains: education and social sciences (212 male students); applied and technological sciences (186 students); and medical and health sciences (164 students). The range of age was between 18 and 23 ( $\mathrm{M}=$ $21.01, S D=2.99$ ).

As the authors are faculty members in the department of psychology at Umm Alqura University, the ethics approval for the study was obtained prior to data collection, and each participant provided informed consent before completing questionnaires. The collection of data was concluded in the period of 2 February to 27 April 2016. Authors informed participants about the objective of the study and also about their participation which was voluntary. Both oral and written instructions were given in Arabic to make sure that participants understand them (i.e., there was no right or wrong answer to the questions and they should freely express their opinions), and they were also reassured about the confidentiality of their responses.

For both the mindful attention awareness scale (MAAS) and the brief resilience scale (BRS), "forward and backward" translation by four bilingual translators was conducted, as an Arabic version of two scales equivalent to the original were developed. Specifically, the original scales were translated from English into Arabic by two bilingual translators. The translated versions was then again translated into English by two independent translators. To ensure comparability and equivalence, translators were not affiliated directly with the study (Hess, Sénécal, \& Vallerand, 2000). Using the different versions, authors created the Arabic version for both MAAS and BRS. An independent psychologist revised the created versions. In general, for the two scales, minor differences on the two Arabic versions were corrected at this stage by joint agreement between different translations.

\section{Measures}

Mindful attention awareness scale (MAAS). The scale is composed of a 15-item single factor self-report scale designed to assess one's general tendency for acceptance and attention over a period of time. Respondents rate the 
degree to which they function without awareness of present experience in daily life, covering cognitive, emotional, physical and interpersonal dimensions. Items are rated on a 6point Likert scale from 1 (almost always) to 6 (almost never). A sample item is, "I could be experiencing some emotion and not be conscious of it until sometime later". Studies highlight a high internal consistency as Cronbach's alpha range from 0.82 to 0.87 , and good test-retest reliability (Schmertz, Anderson, \& Robins, 2009). Consequently, the original MAAS was used in this research. Total scores range from 15 to 90, with higher scores reflecting greater mindfulness (Brown \& Ryan, 2003).

Brief Resilience Scale (BRS). This scale is composed of six items. Items 1,3 , and 5 are positively worded, and items 2, 4, and 6 are negatively worded. Sample items are, "I tend to bounce back quickly after hard times," and "I have a hard time making it through stressful events (R)". The BRS was scored by reverse coding items 2, 4, and 6 and finding the mean of the six listed items. Items were rated on a 5point Likert scale ranging from 1 (strongly disagree) to 5 (strongly agree). Studies highlighted a high internal consistency as Cronbach's alpha range from 0.80 to 0.91 (Smith et al., 2008).

\section{Statistical analysis}

Statistical analyses were performed by using SPSS software (v. 22), except the confirmatory factor analyses (CFA) that was conducted with LISREL software (v. 8.8). All data were checked for univariate and multivariate normality. Missing values (representing $1 \%$ of the total data file) were replaced using a regression imputation procedure. The CFA framework provided a means to test the factor structure of the two scales as an important contribution to construct validation in different languages and cultures.

Confirmatory Factor Analyses (CFA). First, we tested the internal consistency calculating the Cronbach's coefficients. Values of 0.70 or greater were considered satisfactory. Then, we performed the CFA to assess the factor to structure both scales. The purpose was to validate if the model fits well with the data. There are many propositions in the literature about the number, type, and cut-off values for goodness-of-fit required to be reported for CFA
(Raju, Laffitte, \& Byrne, 2002). A popular recommendation is to present three of four indices from different areas. Accordingly, we report several goodness-of-fit indicators including GFI (goodness-of-fit index), NNFI (nonnormed fit index), and RMSEA (root mean square error of approximation). The recommended cut-off values for acceptable values are $\geq 0.90$ for GFI and NNFI. The RMR and RMSEA test the fit of the model to the covariance matrix. As a guideline, values below 0.05 indicate a close fit and values below 0.08 are an acceptable fit. Because of that report was based on chi-square value which is limited due to, sample size, we used the chi-square devised on the degree of freedom.

\section{Results}

In the present study, the data were screened for non-normality and no problematic trend was found. To ensure univariate normality, Kline (2005) suggested a cut-off of absolute values of 3.0 and 8.0 for skewness and kurtosis, respectively. For the MAAS, univariate, skewness ranged from -1.33 to 1.16 , and univariate kurtosis ranged from 1.84 to -1.23 , indicating that the responses were relatively normally distributed. We then tested the CFA for the Arabic version of both MAAS (15 items) and BRS (6 items). For RBS, univariate skewness ranged from -0.98 to 0.77 , and univariate kurtosis ranged from 1.17 to -1.23 , indicating that the responses were relatively normally distributed.

For MAAS, results of CFA revealed a satisfactory fit to the data in terms of $\chi 2, d f$ ratio, GFI, NNFI, and RMSEA. The $\chi 2(679.18, \mathrm{~N}=562)=$ $9,35, p=0.002, \mathrm{NNFI}=0.95, \mathrm{CFI}=0.95$, RMSEA $=.05[.04-.06]$, GFI $=0.96$, AGFI $=$ 0.93 . The goodness of fit was acceptable in terms of $x^{2} / d f$ ratio, GFI, NNFI, RMR, and RMSEA (see Table 1). The reliability of the scale was assessed using Cronbach's Alpha. Based on data from the present investigation, it was acceptable $(0.81)$. These results are similar to those found in other studies in different cultures and countries (Black, Sussman, Johnson, \& Milam, 2012; Carlson \& Brown, 2005; Jermann et al., 2009; MacKillop \& Anderson, 2007). 
Table 1

The Correlations between the 15 Items Composing the MAAS $(\mathrm{N}=562)$

\begin{tabular}{|c|c|c|c|c|c|c|c|c|c|c|c|c|c|c|c|c|}
\hline $\begin{array}{c}\text { ite } \\
\mathrm{m}\end{array}$ & 1 & 2 & 3 & 4 & 5 & 6 & 7 & 8 & 9 & 10 & 11 & 12 & 13 & 14 & $\mathrm{M}$ & $S D$ \\
\hline 1 & - & & & & & & & & & & & & & & 5.21 & 1.23 \\
\hline 2 & $0.56^{*}$ & - & & & & & & & & & & & & & 5.24 & 1.25 \\
\hline 3 & $0.47^{*}$ & $0.53^{*}$ & - & & & & & & & & & & & & 5.03 & 1.43 \\
\hline 4 & $0.50^{*}$ & $0.45^{*}$ & $0.49^{*}$ & - & & & & & & & & & & & 5.32 & 1.28 \\
\hline 5 & $0.43^{*}$ & $0.54^{*}$ & $0.47^{*}$ & $0.53^{*}$ & - & & & & & & & & & & 4.99 & 1.45 \\
\hline 6 & $0.47^{*}$ & $0.42^{*}$ & $0.44^{*}$ & $0.46^{*}$ & $0.43^{*}$ & - & & & & & & & & & 4.87 & 1.45 \\
\hline 7 & $0.43^{*}$ & $0.39^{*}$ & $0.46^{*}$ & $0.47^{*}$ & $0.33^{*}$ & $0.46^{*}$ & - & & & & & & & & 4.90 & 1.27 \\
\hline 8 & $0.42^{*}$ & $0.43^{*}$ & $0.49^{*}$ & $0.44^{*}$ & $0.33^{*}$ & $0.44^{*}$ & $0.52^{*}$ & - & & & & & & & 5.02 & 1.37 \\
\hline 9 & $0.38^{*}$ & $0.40^{*}$ & $0.53^{*}$ & $040^{*}$ & $0.45^{*}$ & $0.43^{*}$ & $0.57^{*}$ & $0.43^{*}$ & - & & & & & & 5.06 & 1.49 \\
\hline 10 & $0.33^{*}$ & $0.48^{*}$ & $0.45^{*}$ & $0.39^{*}$ & $0.53^{*}$ & $035^{*}$ & $0.45^{*}$ & $0.54^{*}$ & $0.54^{*}$ & - & & & & & 5.31 & 1.63 \\
\hline 11 & $0.42^{*}$ & $0.47^{*}$ & $0.37^{*}$ & $0.37^{*}$ & $0.46^{*}$ & $0.39^{*}$ & $0.33^{*}$ & $0.44^{*}$ & $0.43^{*}$ & $0.47^{*}$ & - & & & & 5.00 & 1.34 \\
\hline 12 & $0.41^{*}$ & $0.48^{*}$ & $0.38^{*}$ & $0.49^{*}$ & $0.46^{*}$ & $0.35^{*}$ & $0.48^{*}$ & $0.47^{*}$ & $0.42^{*}$ & $0.57^{*}$ & $0.43^{*}$ & - & & & 5.30 & 1.61 \\
\hline 13 & $0.40^{*}$ & $0.48^{*}$ & $0.42^{*}$ & $0.45^{*}$ & $0.38^{*}$ & $0.43^{*}$ & $0.46^{*}$ & $0.49^{*}$ & $0.49^{*}$ & $0.48^{*}$ & $0.48^{*}$ & $0.54^{*}$ & - & & 5.19 & 1.50 \\
\hline 14 & $0.43^{*}$ & $0.49^{*}$ & $0.44^{*}$ & $0.38^{*}$ & $0.36^{*}$ & $0.56^{*}$ & $0.49^{*}$ & $0.47^{*}$ & $0.56^{*}$ & $0.47^{*}$ & $0.42^{*}$ & $0.43^{*}$ & $0.51^{*}$ & - & 5.04 & 1.45 \\
\hline 15 & $0.47^{*}$ & $0.42^{*}$ & $0.45^{*}$ & $0.37^{*}$ & $0.37^{*}$ & $0.55^{*}$ & $0.46^{*}$ & $0.41^{*}$ & $0.49^{*}$ & $0.41^{*}$ & $0.38^{*}$ & $0.45^{*}$ & $0.37^{*}$ & $0.43^{*}$ & 4.73 & 1.55 \\
\hline
\end{tabular}

Table 2

The Correlations between the 6 Items Composing the BRS

\begin{tabular}{|c|c|c|c|c|c|c|c|c|}
\hline item & 1 & 2 & 3 & 4 & 5 & 6 & $\mathrm{M}$ & $S D$ \\
\hline 1 & - & & & & & & 4.68 & 1.65 \\
\hline 2 & $0.33^{*}$ & - & & & & & 4.78 & 1.42 \\
\hline 3 & $0.43^{*}$ & $0.49^{*}$ & - & & & & 4.01 & 1.78 \\
\hline 4 & $0.39^{*}$ & $0.43^{*}$ & $0.51^{*}$ & - & & & 4.82 & 1.39 \\
\hline 5 & $0.49^{*}$ & $0.44^{*}$ & $0.52^{*}$ & $0.49^{*}$ & - & & 4.14 & 1.99 \\
\hline 6 & $0.39^{*}$ & $0.42^{*}$ & $0.53^{*}$ & $0.44^{*}$ & $0.47^{*}$ & - & 4.07 & 1.42 \\
\hline
\end{tabular}

Notes. $n=562 ; 2,4$, 6reverse coded items.

${ }^{*} p<.01$

Concerning the BRS, univariate skewness ranged from -0.92 to 0.88 , and univariate kurtosis ranged from -1.07 to 1.18 , indicating that the responses were relatively normally distributed. Results of CFA revealed a satisfactory fit to the data in terms of $\chi 2$, df ratio, GFI, NNFI, and RMSEA. The $\chi 2(25.27, \mathrm{~N}=562)=9$, $\mathrm{p}=0.00, \mathrm{NNFI}=0.96, \mathrm{CFI}=0.95, \mathrm{RMSEA}=.01$ $[.02 ; .03], \mathrm{GFI}=0.98$, AGFI $=0.96$. The Goodness of Fit was acceptable in terms of $\chi 2 / \mathrm{df}$ ratio, GFI, NNFI, RMR, and RMSEA (table 2). The reliability of the scale was assessed using Cronbach's Alpha. Results based on data findings from the present investigation, it was acceptable by this margin (0.73).

\section{Discussion}

The present research aimed to explore the psychometric proprieties for the Arabic version of the MAAS and BRS, the result confirms the structural validity and reliability of the two scales as a unidimensional construct. These results are similar to the findings of recent studies in many cultures (Amat, Subhan, Jaafar, Mahmud, \& Johari, 2014; RodríguezRey, Alonso-Tapia, \& Hernansaiz-Garrido, 2016; Smith, Epstein, Ortiz, Christopher, \& Tooley, 2013). We concluded that the Arabic version of both scales are unidimensional and have an acceptable reliability as well as the original version.

\section{Study 2}

The goals of the second study are to test a model that describes a mediator role of resilience and flourishing in the relationships between mindfulness as independent variables and life satisfaction score as a dependent variable.

\section{Method}

\section{Participants and procedure}

Like the first study, ethics approval for the study was obtained prior to data collection, and informed consent was provided by each participant prior to completing questionnaires. 
Students were all from the Abidiyyah campus. They studied in one of six colleges: education, social sciences, applied sciences, computer and information systems, medicine and applied medical science. The sample was also randomly selected in the same three different domains as study 1 . They were not the same students that participated in the first study. The collection of data was in the period of 2 February to 27 April 2017. Authors informed participants about the objective of the study and also about their participation which was voluntary. Data collection was administered at a university to unpaid, voluntary students who signed a consent form. They were 534 students in the same three domains as in the previous study. They were aged from 17 to $29(\mathrm{M}=24.03, S D=$ 4.17). The remaining students either did not wish to participate or did not show their interest in the second phase of the study.

Ethics approval of the study was obtained prior to data collection, and each participant provided informed consent prior to completing questionnaires. Authors informed sample about the objective of the study, their participation was voluntary and they could withdraw at any time. The respondents were invited to fill the questionnaire by authors on a voluntary basis. The process of doing the questionnaire tool each respondent about 15 minutes.

\section{Measures}

Mindful Attention Awareness Scale (MAAS). It is composed of a 15-item, single factor selfreport scale designed to assess one's general tendency for acceptance and attention over time. The scale used in the first study.

The Brief Resilience Scale, (BRS). The (BRS) has six items. The scale used in the first study.

The Flourishing Scale (PS). The Flourishing Scale (FS) is a measure of psychosocial flourishing that complements existing measures of subjective well-being. Authors created this brief measure to capture major aspects of this type of "prosperity" based on recent psychological theories of human flourishing. Earlier humanistic psychology theories (Ryff \& Keyes, 1995; Ryff \& Singer, 1998) suggest that there are several universal human psychological needs, such as the need for competence, relatedness, and self-acceptance. Several of these characteristics are assessed by the Flourishing Scale (Diener, Wirtz, et al., 2010). It is a brief 8- item summary measure of respondents selfperceived success in important areas such as relationships, self-esteem, purpose, and optimism. The scale provides a single psychological well-being score (Diener, Wirtz, et al., 2010). The scale shows good psychometric properties in many cultures (L. Hone, Jarden, \& Schofield, 2014; Silva \& Caetano, 2013). The flourishing scale was translated and validated with an Arabic sample (Salama-Younes, 2017).

The Satisfaction with Life Scale (SWLS). The SWLS is a global measure of life satisfaction. The SWLS consists of five items. A 7-point Likert scale was used ranging from "strongly disagree" (1-point) to "strongly agree" (7point). The SWLS is a measure of life satisfaction developed by Diener et al. (1985). The SWLS has good psychometric properties in different cultures (Durak, Senol-Durak, \& Gencoz, 2010; Glaesmer, Grande, Braehler, \& Roth, 2011; Gouveia, Milfont, Da Fonseca, \& de Miranda Coelho, 2009). The scale was translated and validated in an Arabic speaking sample (Abdallah, 1998).

\section{Statistical analysis}

Data was analyzed using SPSS software (v.22) and LISREL Software (v. 8.8).

\section{Results}

In the present research, a positive and significant correlation among the mindfulness, resilience, flourishing and life satisfaction was obtained. It ranged between $r=0.37$ and $0.55, p<$ 0.01 (table 3). For mindfulness, resilience and life satisfaction, findings are similar to those found in the results of previous studies (Bajaj \& Pande, 2016). Based on the existing literature, it is shown that mindfulness is an antecedent to resilience (Pidgeon \& Keye, 2014; Roeser, 2014; Rogers, 2013). According to Ryff, Love, Essex, and Singer (1998) resilience can be seen as an important source of subjective well-being. There is strong evidence that resilience is of considerable benefit to people's subjective well-being. Mindfulness is closely associated with a higher level of well-being (Bajaj, Gupta, \& Pande, 2016; Brown \& Ryan, 2003; Howell, Digdon, Buro, \& Sheptycki, 2008). 
Table 3

The Correlations among the Study Variables

\begin{tabular}{lcccccc}
\hline \multicolumn{1}{c}{ Variable } & Mindfulness & Resilience & Flourishing & Life Satisfaction & $\mathrm{M}$ & $S D$ \\
\hline Mindfulness & --- & & & & 5.11 & 1.36 \\
Resilience & $0.36^{*}$ & -- & & & 4.78 & 1.43 \\
Flourishing & $0.38^{*}$ & $0.36^{*}$ & --- & 4.44 & 1.22 \\
& & & & & \\
Life Satisfaction & $0.49^{*}$ & $0.53^{*}$ & $0.57^{*}$ & ---- & 6.89 & 1.32 \\
\hline $\mathrm{N}=534 ;{ }^{*} \mathrm{p}<.01$ & & & & & &
\end{tabular}

The study tested the hypothesis by which the relationship between mindfulness and life satisfaction was mediated by resilience and flourishing. In accordance with the four steps recommended by Baron and Kenny (1986), data confirm the existence of mediation if (a) the independent variables (i.e., mindfulness) significantly predict the dependent variable (i.e., life satisfaction), (b) the independent variables significantly predict the potential mediator (i.e., resilience and flourishing), and (c) the potential mediator(s) significantly predicts the dependent variable when the effect of the independent variables is controlled by any means. If the independent variables no longer predict the dependent variable when the effect of the mediator is controlled, there is total mediation, whereas there is only partial mediation if the independent variables predicts the dependent variable. Table 3 contains the means, standard deviations, and correlations between the 4 variables. The hypothesis of model 1 stated that resilience should mediate the relationship between mindfulness and flourishing; the flourishing should also mediate the relationships between resilience and life satisfaction. The correlation matrix served as the database for the path analysis and the method of estimation was the maximum likelihood. All the path analyses conducted in the present research were performed with SIMPLIS project.

The present model was composed of one exogenous variable (mindfulness) and three endogenous variables (resilience, flourishing and life satisfaction). Only life satisfaction is a dependent variable. The paths were drawn according to the hypothesis presented above.

Concerning model 1 , result of the path analysis revealed a non-satisfactory fit to the data in terms of $x 2 / d f$ ratio, GFI, NNFI, RMR, and RMSEA. The $\chi 2(1, N=534), \mathrm{NNFI}=0.69, \mathrm{CFI}$ $=0.93$, RMSEA $=0.24, \mathrm{GFI}=0.92, \mathrm{AGFI}=0.73$, $\mathrm{RMR}=0.08$. a shown in figure 1 .

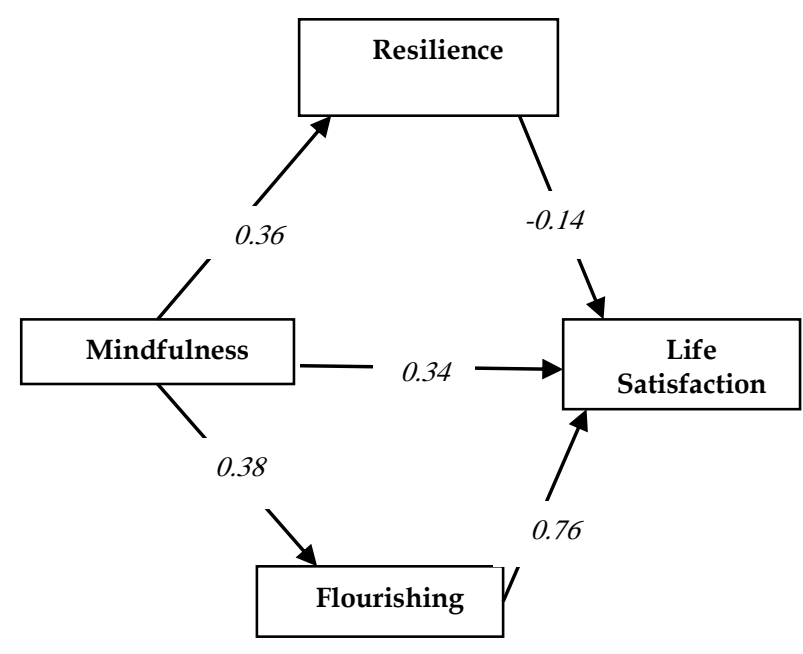

Figure 1: Model 1 in which both of resilience and flourishing is a partial mediator in the relationships between mindfulness and life satisfaction.

Standardized path coefficients are presented Significant direct and indirect effect paths are shown for mindfulness and life satisfaction. However, the values of the path coefficients are those of the full mediating model, including the direct effects of the independent variables to the outcome variables. ${ }^{* *} \mathrm{p} .01$.

Regarding model 2, the hypothesis stated that resilience and flourishing should mediate the relation between mindfulness and life satisfaction. The correlation matrix served as the database for the path analysis and the method of estimation was the maximum likelihood among all variables. All the path analyses conducted in the present research were performed with SIMPLIS project. Although the proposed model among the four constructs has not yet been studied, we tested a model proposed from the software to add a path from resilience to flourishing. A result of the model is saturated and the fit is perfect. All estimated paths were significant at $p=0.01$. According to Souri and Hasanirad (2011), resilience is able to predict psychological wellbeing and their results are similar to our findings. 


\section{Discussion}

Starting from the first aim of this study, findings confirmed the unidimensionality for both MAAS and BRS. Similar result, from many countries confirm that both scales was unidimensional (Brown, West, Loverich, \& Biegel, 2011; Jermann et al., 2009; Newton-John, Mason, \& Hunter, 2014). In the light of previous studies, the first objective of the present research aims to test the dimensionality of the two scales for an Arab population and especially with Saudian samples as collected data also confirmed this hypothesis. For MAAS and BRS, results of CFA revealed a satisfactory fit to the data in terms of $\chi 2$, df ratio, GFI, NNFI and RMSEA. The reliability of the MAAS and BRS were assessed by using Cronbach's Alpha. Based on data from the first study, it was found acceptable (0.83 and 0.78) respectively.

In the second study, the correlation matrix shows good relationships between mindfulness, resilience, and flourishing and life satisfaction. It means that mindfulness could probably lead to equally high levels of resilience, flourishing and life satisfaction (table 3). Mindfulness may facilitate well-being through self-regulated activity and fulfillment of the basic psychological needs for autonomy (selfendorsed or freely chosen activity), competence, and relatedness (Hodgins \& Knee, 2002). Many results have shown that people who are flourishing are more likely to graduate from college, secure "better" jobs, and are more likely to succeed in that job. One reason for this success can be seen in the evidence offered above when discussing languishing: those that flourish have less work absenteeism. Previous research indicated the relationships among mindfulness, resilience, wellbeing and life satisfaction. This relationship is not among the 4 variables is not a causal relationship. For that, we aimed to test two path analysis models in order to explore this relation. The finding indicated that only the second model shows a perfect fit of data in which both of resilience and flourishing are mediator variables. In addition, both resilience and flourishing are partial mediators between mindfulness and life satisfaction. More precisely, partial mediation maintains resilience and flourishing account, for some but not all, of the relationship between the mindfulness and life satisfaction. Partial mediation implies that there is not only a significant rela- tionship between both a resilience, flourishing and life satisfaction, but also some direct relationship between mindfulness and life satisfaction is also exists or present.

Some limitations should be kept in mind when interpreting the current findings. The second study had, at least, two important limitations. First, it is impossible to determine the directionality of causality with respect to the proposed model. Resilience could lead to wellbeing and so why couldn't well-being lead to resilience, for example? Consequently, researchers should try to replicate the present findings using experimental designs in order to clearly establish the directionality of effects. This experimental design should be in a sequence of times. Another limitation would be that this drawback did not permit us to investigate the issue of gender differences and academic fields (scientific vs literature). Future researches should look into this issue given that gender differences have been reported in the mindfulness not only as a cause but as a mediator variable. Mindfulness may also affect one's physical, mental health and emotions. A longitudinal design and mindfulness intervention is one of the gaps identified in this research and also to study its effect on resilience, flourishing and life satisfaction.

\section{References}

Abdallah, T. (1998). The Satisfaction with Life Scale (SWLS): Psychometric properties in an Arabic-speaking sample. International journal of Adolescence and Youth, 7(2), 113119.

Abolghasemi, A., \& Varaniyab, S. T. (2010). Resilience and perceived stress: Predictors of life satisfaction in the students of success and failure. Procedia-Social and Behavioral Sciences, 5, 748-752.

Akin, A., \& Akin, U. (2015). Mediating role of coping competence on the relationship between mindfulness and flourishing. Suma Psicológica, 22(1), 37-43.

Amat, S., Subhan, M., Jaafar, W. M. W., Mahmud, Z., \& Johari, K. S. K. (2014). Evaluation and psychometric status of the brief resilience scale in a sample of Malaysian international students. Asian Social Science, 10(18), 240. 
Aspinwall, L. G., \& MacNamara, A. (2005). Taking positive changes seriously. Cancer, 104(S11), 2549-2556.

Baer, R. A., Smith, G. T., Hopkins, J., Krietemeyer, J., \& Toney, L. (2006). Using self-report assessment methods to explore facets of mindfulness. Assessment, 13(1), 2745.

Bajaj, B., Gupta, R., \& Pande, N. (2016). Selfesteem mediates the relationship between mindfulness and well-being. Personality and Individual Differences, 94, 96-100.

Bajaj, B., \& Pande, N. (2016). Mediating role of resilience in the impact of mindfulness on life satisfaction and affect as indices of subjective well-being. Personality and Individual Differences, 93, 63-67.

Barnes, S., Brown, K. W., Krusemark, E., Campbell, W. K., \& Rogge, R. D. (2007). The role of mindfulness in romantic relationship satisfaction and responses to relationship stress. Journal of Marital and Family Therapy, 33(4), 482-500.

Baron, R. M., \& Kenny, D. A. (1986). The moderator-mediator variable distinction in social psychological research: Conceptual, strategic, and statistical considerations. Journal of Personality and Social Psychology, 51(6), 1173.

Black, D. S., Sussman, S., Johnson, C. A., \& Milam, J. (2012). Psychometric assessment of the mindful attention awareness scale (MAAS) among Chinese adolescents. Assessment, 19(1), 42-52.

Brown, K. W., \& Ryan, R. M. (2003). The benefits of being present: mindfulness and its role in psychological well-being. Journal of Personality and Social Psychology, 84(4), 822.

Brown, K. W., West, A. M., Loverich, T. M., \& Biegel, G. M. (2011). Assessing adolescent mindfulness: validation of an adapted Mindful Attention Awareness Scale in adolescent normative and psychiatric populations. Psychological Assessment, 23(4), 1023.

Buetell, N. (2006). Life satisfaction, a sloan work and family encyclopedia entry. Sloan Work-Family. Encyclopedia.
Burns, R. A., Anstey, K. J., \& Windsor, T. D. (2011). Subjective well-being mediates the effects of resilience and mastery on depression and anxiety in a large community sample of young and middleaged adults. Australian $\mathcal{E}$ New Zealand Journal of Psychiatry, 45(3), 240-248.

Carlson, L. E., \& Brown, K. W. (2005). Validation of the Mindful Attention Awareness Scale in a cancer population. Journal of Psychosomatic Research, 58(1), 2933.

Carmody, J., \& Baer, R. A. (2008). Relationships between mindfulness practice and levels of mindfulness, medical and psychological symptoms and wellbeing in a mindfulness-based stress reduction program. Journal of behavioral Medicine, 31(1), 23-33.

Catalano, D., Chan, F., Wilson, L., Chiu, C.-Y., \& Muller, V. R. (2011). The buffering effect of resilience on depression among individuals with spinal cord injury: a structural equation model. Rehabilitation psychology, 56(3), 200.

Chavers, D. J. (2013). Relationships between spirituality, religiosity, mindfulness, personality, and resilience: University of South Alabama.

Cohrs, J. C., Christie, D. J., White, M. P., \& Das, C. (2013). Contributions of positive psychology to peace: toward global wellbeing and resilience. American Psychologist, 68(7), 590.

Collard, P., Avny, N., \& Boniwell, I. (2008). Teaching mindfulness-based cognitive therapy (MBCT) to students: The effects of MBCT on the levels of mindfulness and subjective well-being. Counselling Psychology Quarterly, 21(4), 323-336.

Collins, S. (2007). Social workers, resilience, positive emotions and optimism. Practice, 19(4), 255-269.

Consten, C. (2016). Measuring resilience with the brief Resilience Scale: Factor Structure, Reliability and Validity of the Dutch Version of the BRS (BRSnl). The University of Twente.

Di Tella, R., Haisken-De New, J., \& MacCulloch, R. (2010). Happiness adaptation to income and to status in an 
individual panel. Journal of Economic Behavior \& Organization, 76(3), 834-852.

Diener, E. (1994). Assessing subjective wellbeing: Progress and opportunities. Social Indicators Research, 31(2), 103-157.

Diener, E., \& Diener, M. (2009). Cross-cultural correlates of life satisfaction and self-esteem Culture and Well-being (pp. 71-91): Springer.

Diener, E., Emmons, R. A., Larsen, R. J., \& Griffin, S. (1985). The satisfaction with life scale. Journal of Personality Assessment, 49(1), 71-75.

Diener, E., Kahneman, D., \& Helliwell, J. (2010). International Differences in Well-being: Oxford University Press.

Diener, E., Wirtz, D., Tov, W., Kim-Prieto, C., Choi, D.-w., Oishi, S., \& Biswas-Diener, R. (2010). New well-being measures: Short scales to assess flourishing and positive and negative feelings. Social Indicators Research, 97(2), 143-156.

Durak, M., Senol-Durak, E., \& Gencoz, T. (2010). Psychometric properties of the satisfaction with life scale among Turkish university students, correctional officers, and elderly adults. Social Indicators Research, 99(3), 413-429.

Fortney, L., Luchterhand, C., Zakletskaia, L., Zgierska, A., \& Rakel, D. (2013). Abbreviated mindfulness intervention for job satisfaction, quality of life, and compassion in primary care clinicians: a pilot study. The Annals of Family Medicine, 11(5), 412-420.

Fredrickson, B. L., \& Losada, M. F. (2005). Positive affect and the complex dynamics of human flourishing. American Psychologist, 60(7), 678.

Frey, B. S., \& Stutzer, A. (2002). What can economists learn from happiness research? Journal of Economic Literature, 40(2), 402-435.

Frijters, P., Johnston, D. W., \& Shields, M. A. (2011). Life satisfaction dynamics with quarterly life event data. The Scandinavian Journal of Economics, 113(1), 190-211.

Glaesmer, H., Grande, G., Braehler, E., \& Roth, M. (2011). The German version of the satisfaction with life scale (SWLS). European Journal of Psychological Assessment.
Gouveia, V. V., Milfont, T. L., Da Fonseca, P. N., \& de Miranda Coelho, J. A. P. (2009). Life satisfaction in Brazil: Testing the psychometric properties of the satisfaction with life scale (SWLS) in five Brazilian samples. Social indicators research, 90(2), 267.

Haidt, J., \& Keyes, C. L. (2003). Flourishing: Positive psychology and the life well-lived: American Psychological Association.

Hess, U., Sénécal, S., \& Vallerand, R. (2000). Les méthodes quantitative et qualitative de recherche en psychologie. RJ, Vallerand et U., Hess (Eds.). Méthodes de recherche en psychologie. Boucherville: Gaëtan Morin.

Hodgins, H. S., \& Knee, C. R. (2002). The integrating self and conscious experience. Handbook of self-Determination Research, 87100.

Hone, L., Jarden, A., \& Schofield, G. (2014). Psychometric properties of the Flourishing Scale in a New Zealand sample. Social indicators research, 119(2), 1031-1045.

Hone, L. C., Jarden, A., Schofield, G. M., \& Duncan, S. (2014). Measuring flourishing: The impact of operational definitions on the prevalence of high levels of wellbeing. International Journal of Wellbeing, 4(1).

Howell, A. J., Digdon, N. L., Buro, K., \& Sheptycki, A. R. (2008). Relations among mindfulness, well-being, and sleep. Personality and Individual Differences, 45(8), 773-777.

Hu, T., Zhang, D., \& Wang, J. (2015). A metaanalysis of the trait resilience and mental health. Personality and Individual Differences, $76,18-27$

Hülsheger, U. R., Alberts, H. J., Feinholdt, A., \& Lang, J. W. (2013). Benefits of mindfulness at work: The role of mindfulness in emotion regulation, emotional exhaustion, and job satisfaction. Journal of Applied Psychology, 98(2), 310.

Huppert, F. A., \& So, T. T. (2013). Flourishing across Europe: Application of a new conceptual framework for defining wellbeing. Social Indicators Research, 110(3), 837861.

Jermann, F., Billieux, J., Larøi, F., d'Argembeau, A., Bondolfi, G., Zermatten, A., \& Van der Linden, M. (2009). Mindful 
Attention Awareness Scale (MAAS): Psychometric properties of the French translation and exploration of its relations with emotion regulation strategies. Psychological Assessment, 21(4), 506.

Jha, A. P., Stanley, E. A., Kiyonaga, A., Wong, L., \& Gelfand, L. (2010). Examining the protective effects of mindfulness training on working memory capacity and affective experience. Emotion, 10(1), 54.

Kabat-Zinn, J. (2009). Wherever you go, there you are Mindfulness Meditation in Everyday Life: Hachette UK.

Keyes, C. L. (2010). Flourishing: Wiley Online Library.

Kline, T. (2005). Psychological testing: A practical approach to design and evaluation: Sage.

Kong, F., Wang, X., Hu, S., \& Liu, J. (2015). Neural correlates of psychological resilience and their relation to life satisfaction in a sample of healthy young adults. Neuroimage, 123, 165-172.

Langer, E. J., \& Moldoveanu, M. (2000). The construct of mindfulness. Journal of social Issues, 56(1), 1-9.

Lightsey Jr, O. R. (2006). Resilience, meaning, and well-being. The Counseling Psychologist, 34(1), 96-107.

Lu, L. (2010). Chinese well-being Oxford handbook of Chinese psychology.

MacKillop, J., \& Anderson, E. J. (2007). Further psychometric validation of the mindful attention awareness scale (MAAS). Journal of Psychopathology and Behavioral Assessment, 29(4), 289-293.

Mak, W. W., Ng, I. S., \& Wong, C. C. (2011). Resilience: enhancing well-being through the positive cognitive triad. Journal of Counseling Psychology, 58(4), 610.

Mankhreh, A., \& Yahya, A. B. (2017). Resiliance and its relation with happiness among addicts and non addicts: A predictive and comaparative stuy.

Markovitz, S. E., Schrooten, W., Arntz, A., \& Peters, M. L. (2015). Resilience as a predictor of emotional response to the diagnosis and surgery in breast cancer patients. Psycho-Oncology, 24(12), 1639-1645.
Martin, J. R. (1997). Mindfulness: A proposed common factor. Journal of Psychotherapy Integration, 7(4), 291.

Matos, P. S., Neushotz, L. A., Griffin, M. T. Q., \& Fitzpatrick, J. J. (2010). An exploratory study of resilience and job satisfaction among psychiatric nurses working in inpatient units. International Journal of Mental Health Nursing, 19(5), 307-312.

Montero-Marin, J., Tops, M., Manzanera, R., Demarzo, M. M. P., de Mon, M. Á., \& García-Campayo, J. (2015). Mindfulness, resilience, and burnout subtypes in primary care physicians: the possible mediating role of positive and negative affect. Frontiers in Psychology, 6.

Newton-John, T. R., Mason, C., \& Hunter, M. (2014). The role of resilience in adjustment and coping with chronic pain. Rehabilitation Psychology, 59(3), 360.

Ong, A. D., Bergeman, C., Bisconti, T. L., \& Wallace, K. A. (2006). Psychological resilience, positive emotions, and successful adaptation to stress in later life. Journal of Personality and Social Psychology, 91(4), 730.

Pidgeon, A. M., \& Keye, M. (2014). Relationship between resilience, mindfulness, and pyschological well-being in University students. International Journal of Liberal Arts and Social Science, 2(5), 27.

Portzky, M., Wagnild, G., De Bacquer, D., \& Audenaert, K. (2010). Psychometric evaluation of the Dutch Resilience Scale RS$\mathrm{nl}$ on 3265 healthy participants: a confirmation of the association between age and resilience found with the Swedish version. Scandinavian Journal of Caring Sciences, 24(s1), 86-92.

Prasoon, R., \& Chaturvedi, K. (2016). Life Satisfaction: A Literature Review. International Journal of Management Humanities and Social Sciences, 1(2), 25-32.

Raju, N. S., Laffitte, L. J., \& Byrne, B. M. (2002). Measurement equivalence: A comparison of methods based on confirmatory factor analysis and item response theory. Journal of Applied Psychology, 87(3), 517.

Rodríguez-Rey, R., Alonso-Tapia, J., \& Hernansaiz-Garrido, H. (2016). Reliability 
and validity of the Brief Resilience Scale (BRS) Spanish Version. Psychological Assessment, 28(5), e101.

Roeser, R. W. (2014). The emergence of mindfulness-based interventions in educational settings Motivational Interventions (pp. 379-419): Emerald Group Publishing Limited.

Rogers, H. B. (2013). Mindfulness meditation for increasing resilience in college students. Psychiatric Annals, 43(12), 545-548.

Ryff, C. D., \& Keyes, C. L. M. (1995). The structure of psychological well-being revisited. Journal of Personality and Social Psychology, 69(4), 719.

Ryff, C. D., Love, G. D., Essex, M. J., \& Singer, B. (1998). Resilience in adulthood and later life Handbook of aging and mental health (pp. 69-96): Springer.

Ryff, C. D., \& Singer, B. (1998). The contours of positive human health. Psychological inquiry, 9(1), 1-28.

Salama-Younes, M. (2017). Psychometric properties of the Psychological Flourishing Scale in an Egyptian setting. Journal of Psychology in Africa, 27(4), 310-315.

Schmertz, S. K., Anderson, P. L., \& Robins, D. L. (2009). The relation between self-report mindfulness and performance on tasks of sustained attention. Journal of Psychopathology and Behavioral Assessment, 31(1), 60-66.

Schotanus-Dijkstra, M., Pieterse, M. E., Drossaert, C. H., Westerhof, G. J., De Graaf, R., Ten Have, M., . . . Bohlmeijer, E. T. (2016). What factors are associated with flourishing? Results from a large representative national sample. Journal of Happiness Studies, 17(4), 1351-1370.

Schueller, S. M., \& Parks, A. C. (2014). The science of self-help. European Psychologist.

Schutte, N. S., \& Malouff, J. M. (2011). Emotional intelligence mediates the relationship between mindfulness and subjective well-being. Personality and Individual Differences, 50(7), 1116-1119.

Senik, C. (2011). Is happiness different from flourishing? Cross-country evidence from the ESS. Revue D'économie Politique, 121(1), 17-34.
Shapiro, S. L., Oman, D., Thoresen, C. E., Plante, T. G., \& Flinders, T. (2008). Cultivating mindfulness: effects on wellbeing. Journal of Clinical Psychology, 64(7), 840-862.

Silva, A. J., \& Caetano, A. (2013). Validation of the flourishing scale and scale of positive and negative experience in Portugal. Social Indicators Research, 110(2), 469-478.

Smith, B. W., Dalen, J., Wiggins, K., Tooley, E., Christopher, P., \& Bernard, J. (2008). The brief resilience scale: assessing the ability to bounce back. International journal of Behavioral Medicine, 15(3), 194-200.

Smith, B. W., Epstein, E. M., Ortiz, J. A., Christopher, P. J., \& Tooley, E. M. (2013). The foundations of resilience: What are the critical resources for bouncing back from stress? Resilience in Children, Adolescents, and Adults (pp. 167-187): Springer.

Souri, H., \& Hasanirad, T. (2011). Relationship between resilience, optimism and psychological well-being in students of medicine. Procedia-Social and Behavioral Sciences, 30, 1541-1544.

Tsaousis, I., Nikolaou, I., Serdaris, N., \& Judge, T. A. (2007). Do the core self-evaluations moderate the relationship between subjective well-being and physical and psychological health? Personality and Individual Differences, 42(8), 1441-1452.

Upadyaya, K., \& Salmela-Aro, K. (2013). Development of school engagement in association with academic success and well-being in varying social contexts. European Psychologist.

Van Breda, A. D. (2001). Resilience theory: A literature review. Pretoria, South Africa: South African Military Health Service.

Van Hoorn, A. (2007). A short introduction to subjective well-being: Its measurement, correlates and policy uses.

Van Zyl, L. E., \& Rothmann, S. (2012). Flourishing of students in a tertiary education institution in South Africa. Journal of Psychology in Africa, 22(4), 593-599.

Veenhoven, R. (1996). Developments in satisfaction-research. Social Indicators Research, 37(1), 1-46. 
Weinstein, N., Brown, K. W., \& Ryan, R. M. (2009). A multi-method examination of the effects of mindfulness on stress attribution, coping, and emotional well-being. Journal of Research In Personality, 43(3), 374-385.

Windle, G., Bennett, K. M., \& Noyes, J. (2011). A methodological review of resilience measurement scales. Health and Quality of Life Outcomes, 9(1), 8.

Wright, T. A., \& Cropanzano, R. (2000). Psychological well-being and job satisfaction as predictors of job performance. Journal of occupational Health Psychology, 5(1), 84. 